\title{
La poétique documentaire de Bouvard et Pécuchet
}

Visibilité des savoirs et arrangement scénarique

Norioki Sugaya

\section{CpenEdition}

Journals

Édition électronique

URL : http://journals.openedition.org/aes/569

DOI : $10.4000 /$ aes.569

ISSN : 2258-093X

Éditeur

Laboratoire LISAA

Référence électronique

Norioki Sugaya, "La poétique documentaire de Bouvard et Pécuchet », Arts et Savoirs [En ligne],

1 | 2012, mis en ligne le 15 février 2012, consulté le 04 mai 2019. URL : http://

journals.openedition.org/aes/569; DOI : 10.4000/aes.569

Ce document a été généré automatiquement le 4 mai 2019.

Centre de recherche LISAA (Littératures SAvoirs et Arts) 


\title{
La poétique documentaire de Bouvard et Pécuchet
}

\author{
Visibilité des savoirs et arrangement scénarique
}

\author{
Norioki Sugaya
}

1 De Madame Bovary à Bouvard et Pécuchet, l'éventail des productions flaubertiennes nous impressionne par son extrême diversité tant thématique qu'esthétique. Les lecteurs sont chaque fois en présence d'un univers distinct édifié sur des ressources narratives propres et qui engendrent des effets spécifiques. Cette richesse de l'art flaubertien coïncide parfaitement avec les principes esthétiques formulés dans la Correspondance. Flaubert écrivait ainsi dans une lettre à Louise Colet du 29 janvier 1854 : « Chaque œuvre à faire a sa poétique en soi, qu'il faut trouver. $»^{1} \mathrm{Du}$ coup, l'écrivain se trouve confronté à des enjeux différents pour chacune de ses entreprises littéraires, qu'il doit chercher à doter d'une spécificité irréductible. Cet impératif que Flaubert mettait soigneusement en pratique, et qui n'est vraisemblablement pas sans rapport avec l'historicité même du roman moderne ${ }^{2}$ , l'amenait nécessairement à un travail d'écriture considérable. Les innombrables pages manuscrites constituant l'avant-texte flaubertien témoignent en effet d'un effort soutenu pour assurer une singularité fondamentale à chaque œuvre romanesque projetée.

Quelle est alors la poétique propre de Bouvard et Pécuchet, roman posthume et inachevé de Flaubert? Pour élucider ce problème, les lecteurs d'aujourd'hui ont la chance de pouvoir se reporter à l'énorme ensemble des manuscrits de l'œuvre que le romancier a laissé après sa mort et qui se trouve actuellement conservé à la Bibliothèque municipale de Rouen. Les deux projets d'édition électronique en cours, l'un concernant les scénarios et les brouillons (ms gg 10 «Plan»; g 224 « Manuscrit autographe »; g 225 «Brouillons ») , et l'autre les dossiers documentaires parmi lesquels se trouvent les notes de lecture prises par le romancier sur différents domaines du savoir aussi bien que les pages préparées pour le second volume (ms g 226 «Recueils de documents divers») ${ }^{4}$, nous donnent maintenant accès à la quasi-totalité de l'avant-texte de l'œuvre. Ces apports sont d'autant plus précieux que le texte de Bouvard pose avec une acuité particulière la question de l'intertextualité et que seule l'étude de la genèse permet de repérer plus ou moins 
systématiquement l'intertexte relevant du champ des connaissances, qui s'avère inséparable de la poétique du roman encyclopédique.

Le dernier roman de Flaubert se caractérise effectivement par son encyclopédisme affiché. Il abonde en références scientifiques explicites, mais recèle encore davantage de sources implicites dont il est souvent impossible pour les lecteurs de connaître l'identité, voire de déceler l'existence. La fiction de Bouvard et Pécuchet repose sur une érudition étendue dont une part seulement est visible dans la version publiée. Pour citer un exemple, au chapitre III, les deux bonshommes, acceptant le défi du docteur Vaucorbeil, décident d'entreprendre l'étude de la physiologie. À cet effet, ils vont jusqu'à Bayeux et se procurent chez un bouquiniste "les traités de Richerand et d'Adelon, célèbres à l'époque » (p. 113)5. Il s'agit des Nouveaux éléments de physiologie de Balthasar-Anthelme Richerand ( $8^{e}$ édition, Paris, Caille et Ravier, 1820, 2 vol. ) et de la Physiologie de l'homme de Nicolas-Philibert Adelon (Paris, Compère jeune, 1823-1824, 4 vol.). Or, l'examen des manuscrits montre que les deux ouvrages font effectivement partie de la liste des lectures préparatoires du roman encyclopédique, mais aussi qu'il existe bien d'autres sources médicales qui ont également servi à la rédaction de l'épisode de la physiologie. On peut alors se demander pourquoi Flaubert n'a retenu que ces deux références dans le texte et en a dissimulé d'autres non moins importantes. Il est vrai que la disparition des noms propres au cours du processus de réécriture est un phénomène général chez Flaubert et ne se limite point à Bouvard. Mais dans ce cas précis, c'est certainement le souci du temps diégétique qui a guidé l'auteur dans le choix des références ${ }^{6}$. Le chapitre de la médecine se situant avant 1848 , les deux personnages ne peuvent pas consulter sans anachronisme des ouvrages comme le Traité élémentaire de physiologie humaine de Jules Béclard (Paris, Labé, 1855) ou Structure et physiologie animales d'Achille Comte (Paris, Masson, 1853), deux sources qui ont pourtant joué un rôle primordial dans la genèse du chapitre.

Le roman encyclopédique pose donc inévitablement le problème de l'intégration des savoirs dans la fiction. L'érudition, dont la majeure partie reste cachée derrière la surface achevée du texte final, se révèle inhérente à la conception même du roman. La documentation immense que Flaubert a accumulée pendant plusieurs années en préparant ce roman n'avait pas seulement pour but de fournir des informations et des détails techniques, mais de servir d'amorce narrative au récit et d'alimenter ainsi l'univers romanesque. Celui-ci, dans le cas de Bouvard et Pécuchet au moins, ne s'est pas construit indépendamment du chantier documentaire. Bien au contraire, l'histoire des deux bonshommes a pris naissance dans ce que Jacques Neefs appelle «l'imaginaire des documents $»^{7}$. C'est la configuration des savoirs, leur disposition et leurs contradictions réciproques qui procurent à la prose narrative son rythme et ses articulations.

Fiction des savoirs, Bouvard et Pécuchet donne forme et visibilité à la part de romanesque comprise dans les discours cognitifs. Du reste, il convient de rappeler que l'œuvre a «la prétention d'être comique ", comme le précise Flaubert dans ses lettres ${ }^{8}$. Ce comique lié directement à la dimension épistémologique, l'écrivain le nomme « le comique d'idées »" Dérivant des configurations discursives que dessinent les énoncés scientifiques, le comique d'idées accorde à la représentation romanesque une certaine efficacité critique. Le rire est une arme, comme on dit fréquemment. Conçu comme «le livre des vengeances $»^{10}$ contre la bêtise de son époque, Bouvard et Pécuchet garde trace de cette origine polémique jusqu'au bout, en dépit de tous les efforts de rédaction visant plutôt à l'effet d'impersonnalité (ou d'indécidabilité selon le terme de la critique moderne). « Encyclopédie critique en farce $~^{11}$ ainsi que le définit son auteur, le roman est donc une 
mise en question radicale des savoirs qu'il dramatise de manière comique. On voit que le projet est presque une gageure. L'auteur est lui-même conscient de la grande difficulté de ce qu'il entreprend et semble même parfois douter de la réussite tout en insistant fortement sur l'originalité profonde du livre : «Il est possible que je m'y noie, mais si je m'en tire, le globe terrestre ne sera pas digne de me porter. $»^{12}$

Après cet aperçu général sur les rapports entre le narratif et l'épistémologique dans Bouvard et Pécuchet, nous allons maintenant regarder de plus près le processus d'élaboration de l'œuvre et tenter de mettre en lumière la " mécanique compliquée " $^{13}$ par laquelle l'écrivain arrive à capter les potentialités narratives des savoirs et à les transformer en figures de fiction. L'aventure des deux bonshommes, naissant de la «surabondance des documents $»^{14}$ et ayant comme matériau le champ encyclopédique, n'offre pas de péripéties ni d'événements extraordinaires et incite à nous interroger sur les limites du genre romanesque. « Pas de monstres, pas de Héros ! », prétend Flaubert qui dit « [s'être] détourné, exprès, de l'Accidentel et du dramatique ${ }^{15}$. Ou plus précisément, le vrai drame dans Bouvard et Pécuchet réside dans les figures de pensée qui forment à elles seules une matière romanesque très féconde. Aussi le travail de l'écrivain porte-t-il principalement sur les théories et les idées qui se rapportent aux différentes disciplines comme l'agriculture, la géologie ou la philosophie. Nous prenons ici comme exemple le chapitre de la médecine, un des chapitres les plus documentés: le dossier des notes se compose de 136 feuillets, soit 233 pages (ms g 2267, fo 24-158). Avec les nombreux brouillons que nous avons transcrits récemment pour l'édition électronique évoquée plus haut, ce chapitre offre par la complétude de son dossier de genèse un cas exemplaire et idéal pour les investigations critiques.

Un examen rapide de l'avant-texte permet de distinguer deux modalités de l'intégration à la diégèse : le dialogue et le tableau. Premièrement, les savoirs sont mis en texte sous forme de dialogue tantôt au style direct, tantôt au style indirect ou encore au style indirect libre. Flaubert emploie parfois aussi le discours narrativisé. Ainsi, des opinions ou positions scientifiques contradictoires sont réparties entre des personnages dont les échanges verbaux vont venir tracer un partage du savoir. En dépit de leur incompétence manifeste, ils représentent alors chacun une doctrine ou une théorie sur un mode éminemment comique. Il arrive également à Flaubert d'insérer des apories du savoir dans le récit qui prend alors la forme d'une espèce de monologue intérieur. Par exemple, lorsque Bouvard et Pécuchet s'occupent de la nutrition, cette «impasse de la physiologie $»^{16}$, les lecteurs ne savent plus distinguer les voix des deux protagonistes, fusionnées pour ainsi dire en une seule énonciation à l'indirect. Des difficultés scientifiques extraites des livres eux-mêmes ${ }^{17}$ se voient greffées sur le récit romanesque et assimilées aux paroles des personnages : «La nutrition les tourmentait. / Comment se fait-il que le même suc produise des os, du sang, de la lymphe et des matières excrémentielles? Mais on ne peut suivre les métamorphoses d'un aliment. [...] » (118).

Du direct à l'indirect libre ou parfois même au direct libre, des formes variées de dialogue constituent autant de ressources narratives susceptibles d'incorporer des discours du savoir dans le contexte de la fiction. Pour y voir plus clair, prenons un exemple typique, celui d'un dialogue au style direct. Il s'agit de la dispute entre Pécuchet et Vaucorbeil qui se livrent, au chevet du fermier Gouy atteint d'une fièvre typhoïde, un combat aussi violent que cocasse. La discussion portant d'abord sur «la nature des fièvres » passe ensuite à la question de la nourriture ${ }^{18}$. Afin de réfuter Pécuchet qui souligne la nécessité de nourrir le malade, le docteur recourt alors à un autre argument : 
- « Jamais! Son pouls donne quatre-vingt-dix-huit pulsations. »

- «Qu'importe les pulsations ! " Et Pécuchet nomma ses autorités. (123)

Arrêtons-nous un moment sur la genèse de ce petit dialogue qui présente plusieurs points intéressants. Son parcours génétique est composé de onze occurrences qui appartiennent toutes, sauf la dernière, au volume $\mathrm{g} 225^{3}$ :
1) $f^{\circ} 213$ 2) fo 301
3) $f^{\circ} 260 v$
4) $f^{\circ} 318$
5) $f^{\circ} 323$
6) $f^{\circ} 313$
7) fo 319
8) fo 321
9) $\mathrm{f}^{\circ} 325 \mathrm{v}-315$
10) $f^{\circ} 357 v$
11) g $224, f^{\circ} 57^{19}$

Tout d'abord, les lecteurs peuvent se demander qui sont au fond les autorités citées par Pécuchet. Dans la version publiée, le texte enchaîne immédiatement sur un autre problème, celui de l'opposition entre la théorie et la pratique (" "Laissons les systèmes !" dit le docteur »), tandis que dans les brouillons sont donnés les noms de ces autorités : " car ils possédaient maintenant toute une bibliothèque : Eapuron, Tissot, la pathologie d'Andral, etc. » $\left(f^{\circ} 315\right)^{20}$. Ces trois références ${ }^{21}$, associées à la bibliothèque des deux protagonistes, ont été réellement lues par Flaubert, qui a même pris des notes. Par conséquent, il serait légitime de supposer qu'elles constituent les sources de ce passage, d'autant que leur mention n'est barrée qu'au tout dernier moment (manuscrit autographe: g 224, fo 57 ) Or, il n'en est rien. En fait, c'est l'article «Occulte» du Dictionnaire des sciences médicales qui est à son origine, comme le prouvent les notes prises par Flaubert: "La montre à secondes, pour mesurer le pouls, charlatanisme. Car la numération des pulsations ne forme pas le principal caractère du pouls. C'en est à peine un des éléments » $\left(\mathrm{g} 226^{7}, \mathrm{f}^{\circ} 121 \mathrm{v}\right)^{22}$. Cet article de Nacquart semble avoir aussitôt inspiré à Flaubert l'idée d'exposer une divergence d'opinions sur ce sujet dans une scène clinique : «examen des maladies, par pouls, \& langue, trompent et (inutile.)» $\left(f^{\circ} 301\right)^{23}$. Dans la version suivante, l'idée est insérée dans le cadre de l'épisode sur la fièvre typhoïde. Flaubert recopie à peu près tels quels les énoncés des notes de lecture qu'il met maintenant dans la bouche de Pécuchet: "La discussion [...] its disent Pé[cu]chet dit que [...] - montre à secondes, charlatan - le nombre des pulsations n'est pas le principal caractère de la fièvre " (fo $260 \mathrm{v})$. Les brouillons successifs montrent l'écrivain essayant différentes formulations de la réplique de Péuchet, dont voici les occurrences dans l'ordre chronologique :

- Le pouls ne signifie rien - Le nombre des pulsations n'est pas le principal caractère de la fièvre. Dans les apoplexies \& les convulsions des femmes en couches, on trouve souvent les artères fémorales \& céphaliques dans un état inverse, les premières grosses \& gorgées, les secondes vides. ( $f^{\circ} 318$ )

- Le nombre des pulsations ne prouve rien. Dans les apoplexies on trouve très souvent les artères fémorales \& céphaliques dans un état inverse, les premières sont grosses, les secondes vides. (fo 323 )

- Je me moque des pulsations. Elles n'ont pas l'importance qu'on leur croit. (fo 313 )

- Je me moque des pulsations ! Elles n'ont pas d'importance symptomatique. (f॰ 319 )

- Je me moque des pulsations ! Ça ne prouve rien. ( $f^{\circ} 321$ )

- Je me moque des pulsations! ( $f^{\circ} 325 \mathrm{v}$ et fo $357 \mathrm{v}$ )

- Qu'importe les pulsations! (g 224, fo 57)

Le processus génétique qu'on voit ici à l'œuvre est tout à fait exemplaire. Au fo 318 , Flaubert ajoute à l'argument de Nacquart un autre destiné à illustrer également le caractère ambigu du pouls comme preuve. La référence n'en est pas explicitée, mais nous savons qu'il s'agit d'un extrait tiré de l'ouvrage d'Adelon ${ }^{24}$. Les deux arguments, articulés dans les propos de Pécuchet, concourent ici à ôter aux pulsations leur valeur symptomatique. Cependant, après cette étape qui correspond au degré maximal 
d'éclaircissement, l'écriture se fait de plus en plus elliptique. Les discours capables de justifier l'objection de Pécuchet finissent par disparaître complètement au profit d'une simple réaction émotionnelle ( $q u$ 'importe»). Le processus de réduction et de condensation entraîne en même temps un effet de démotivation et rend relativement opaque la réplique du personnage dont la densité épistémologique réelle reste à peine visible pour les lecteurs du texte publié.

Revenons au problème des références. Nous avons constaté tout à l'heure que les auteurs médicaux nommés par Pécuchet dans quelques brouillons ne sont pas les sources du passage. Mais alors, dans quelle intention Flaubert a-t-il inventé de fausses références? Était-ce pour ce qu'il appelle lui-même "[son] but (secret)", c'est-à-dire "ahurir tellement le lecteur qu'il en devienne fou $»^{25}$ ? Nous ne le croyons pas. Ici encore, l'examen des manuscrits nous aide à mettre au jour un phénomène complexe. Contre toute attente, c'est en fait un hasard qui a rattaché ces noms propres à la question des pulsations. Pour s'en convaincre, il faut replacer le dialogue sur le pouls dans un contexte plus large. Ainsi, au fo 323 , la réplique de Pécuchet que nous avons citée plus haut est précédée d'un autre échange :

- Regardez sa langue.

- L'état de la langue est inutile.

- Mais son pouls

On peut considérer qu'à cette étape rédactionnelle, les deux personnages sont censés se disputer sur l'examen médical en général et notamment sur la valeur des signes cliniques. Dans ce contexte, la mention de la bibliothèque qui constitue à elle seule un alinéa ( «Il récitait par cœur des passages - sa bibliothèque, Capuron, Tissot, Buchon -») renvoie plutôt, nous semble-t-il, à la culture médicale générale du personnage qui lui permet de s'opposer au docteur Vaucorbeil sur tel ou tel point de la clinique et ne se rapporte pas uniquement à la question du pouls. En ce sens, il est significatif que les trois auteurs cités (Buchon est un lapsus de Flaubert pour Buchan) soient tous des auteurs de vulgarisation qui ont écrit des manuels de médecine domestique, genre très répandu dans la première moitié $d u \mathrm{XIX}^{\mathrm{e}}$ siècle ${ }^{26}$. Leurs ouvrages ont toutes les raisons de figurer dans la bibliothèque des amateurs qui s'intéressent comme Bouvard et Pécuchet à des acquis scientifiques à leur portée.

Désignant au début l'étendue des connaissances médicales obtenues par les deux autodidactes, la bibliothèque ne tarde toutefois pas à prendre un autre sens. Au fo 323 , l'échange sur l'état de la langue est déjà supprimé et remplacé par un ajout : « Un homme dont le pouls bat X par minutes! » Cette phrase attribuée au médecin se trouve reliée par un trait à la question de la nourriture, ce qui modifie sensiblement son contexte. On n'a ainsi plus affaire à l'examen clinique en général, mais au seul problème de la valeur du pouls. Dans l'occurrence suivante (f $f^{\circ} 313$ ), l'écrivain barre le second argument de Pécuchet relatif à l'état des artères dans les apoplexies, et ajoute en interligne : « \& il cita les autorités». De telles corrections sont fréquentes chez Flaubert, écrivain dont on connaît la tendance à gommer les motivations trop précises et à rendre flous les enchaînements narratifs. Mais cette fois-ci, son art de l'ellipse entraîne un effet sémantique imprévu. De fait, cette petite phrase qui vient clore la réplique de Pécuchet infléchit le sens du paragraphe suivant. Désormais, le lecteur est obligé d'assimiler les noms des auteurs médicaux cités aux « autorités » que Pécuchet allègue dans le but de remettre en cause l'importance des pulsations. Flaubert semble ne s'être aperçu de cet effet gênant que tardivement, car c'est seulement sur le manuscrit définitif ( $g$ 224, fo 57) 
qu'il a éliminé la mention de la bibliothèque et des livres la constituant. Cet exemple de la manipulation de l'intertexte est fort révélateur. Il montre qu'un écrivain aussi consciencieux que Flaubert ne peut pas tout contrôler de la production de son texte et que le processus de rédaction comprend toujours un degré d'incertitude que l'écrivain s'efforce de maîtriser après coup. Parfois, deux notations juxtaposées donnent lieu à une interprétation inattendue dont il appartient à l'auteur d'exploiter ou de rejeter la possibilité. L'invention romanesque, si méthodique soit-elle, n'en affronte pas moins cette part de hasard (qu'on pourrait qualifier de mallarméen), sans doute intrinsèque au dynamisme même de l'écriture.

Passons à l'autre mode d'intégration des savoirs, c'est-à-dire le tableau. Le terme appartient au vocabulaire critique de Flaubert lui-même, et désigne métaphoriquement le caractère visuel de sa représentation romanesque ${ }^{27}$. Dans la Correspondance, il est utilisé soit pour mettre l'accent sur l'impersonnalité de l'art : «Quel est l'esprit un peu fort qui ait conclu, à commencer par Homère ? Contentons-nous du tableau, c'est ainsi, bon $»^{28}$; soit pour exprimer les unités constituantes du récit : «J'ai encore trois petits tableaux à faire, c'est-à-dire 5 à 6 pages environ. $»^{29}$ Dans l'avant-texte des œuvres, le mot figure fréquemment à l'étape des scénarios ou des premiers brouillons, et fait fonction de note de régie indiquant un passage descriptif à élaborer ${ }^{30}$. Ainsi, au fo 213 , la mention du scandale provoqué par le mannequin anatomique («Mannequin. - Scandale. Le maire») est accompagnée de cet ajout interlinéraire : «chez eux - tableau ». Il s'agit là d'une consigne que l'écrivain se donne à lui-même et qui a pour but de programmer l'agencement narratif du texte. Les brouillons du chapitre III comprennent également des formules comme "portrait ", « description », « décrire ", « montrer » ou "développer l'image » qui doivent être comprises comme autant de balises posées pour orienter l'écriture. L'importance accordée à ce métalangage renvoyant à la catégorie du descriptif n'a, du reste, rien de surprenant, «la faculté de faire voir »" étant pour Flaubert une qualité essentielle de l'art romanesque.

16 Pourtant comment faire voir les savoirs ? Voilà assurément une difficulté fondamentale que l'auteur de Bouvard tentait de résoudre, par exemple, en « repren[ant] des notes sur la Physiologie - et la thérapeutique, au point de vue comique ${ }^{32}$. Le travail scénarique de coordination des extraits, qui aboutit à une dizaine de pages de «notes de notes " ( $\mathrm{g} 226^{7}$, $f^{\circ}$ 138-146), tend à la fois à mettre en évidence les contradictions ou les apories et à exploiter les possibilités d'images que renferment les discours médicaux. En un mot, il faut " rendre plastiques $\|^{33}$ les savoirs et leur donner un relief esthétique pour qu'ils puissent entrer dans l'espace de la fiction. Sur ce point, le fo 279 est très significatif ; c'est un scénario développé où on lit, après la courte phrase introductive («Cependant ils firent des expériences »), l'esquisse d'une série d'expériences de physiologie dont quelques-unes se maintiendront jusqu'à la version publiée. Rassemblés ainsi sur une page, ces gestes prétendument scientifiques frappent par leur visibilité burlesque accentuée par la mise en scène des corps des personnages. Nous nous intéressons ici à deux fragments qui, élaborés d'abord séparément, se verront ensuite réunis dans un épisode que l'on peut considérer comme l'un des moments les plus forts du grotesque dans le roman : " - si la contraction sans locomotion développe du Calorique. bain. - agiter l'eau avec les membres - grelotte. froid»; et "restent nus dans une balance. - si le poids du corps diminue par la perspiration. - étudier les changements de poids à l'exemple de Sanctorius ».

Nous nous attarderons un peu sur la genèse de cet épisode qui mérite d'être étudiée attentivement. D'abord, en ce qui concerne la dimension documentaire, l'épisode repose 
sur plusieurs sources médicales qui sont en quelque sorte condensées dans les quelques dizaines de lignes du texte publié. Cette densité épistémologique remarquable peut être constatée par exemple sur un brouillon, fo $270 \mathrm{v}$ (dixième occurrence), où l'on trouve inscrites des références comme "Adelon 3 (ou 4)», "SM peau 18 " (c'est-à-dire le Dictionnaire des sciences médicales, article Peau), «Lévy 2 » et « Béraud 411 » (les chiffres renvoient aux pages de notes de lecture, sauf le dernier exemple où il indique la page de la source). Si l'on examine le dossier des notes médicales, on se rend compte que d'autres sources encore ont contribué à la conception de l'épisode ${ }^{34}$, qui est le produit d'un travail sur l'intertexte particulièrement intense. Néanmoins, l'auteur ne procède pas seulement à la condensation des sources, mais à de nombreuses opérations au nombre desquelles le travail scénarique d'arrangement est de la première importance. Dans les pages qui suivent, nous nous concentrerons sur ce travail de l'écrivain qui, en ajustant les composantes de l'économie narrative, construit des tableaux dont la puissance visuelle provient de l'équilibre péniblement obtenu du récit. Le processus d'élaboration est ici extrêmement complexe, de sorte que le parcours génétique des brouillons $\left(\mathrm{g} 225^{3}\right)$ prend la forme suivante ${ }^{35}$ :

Processus génétique des brouillons

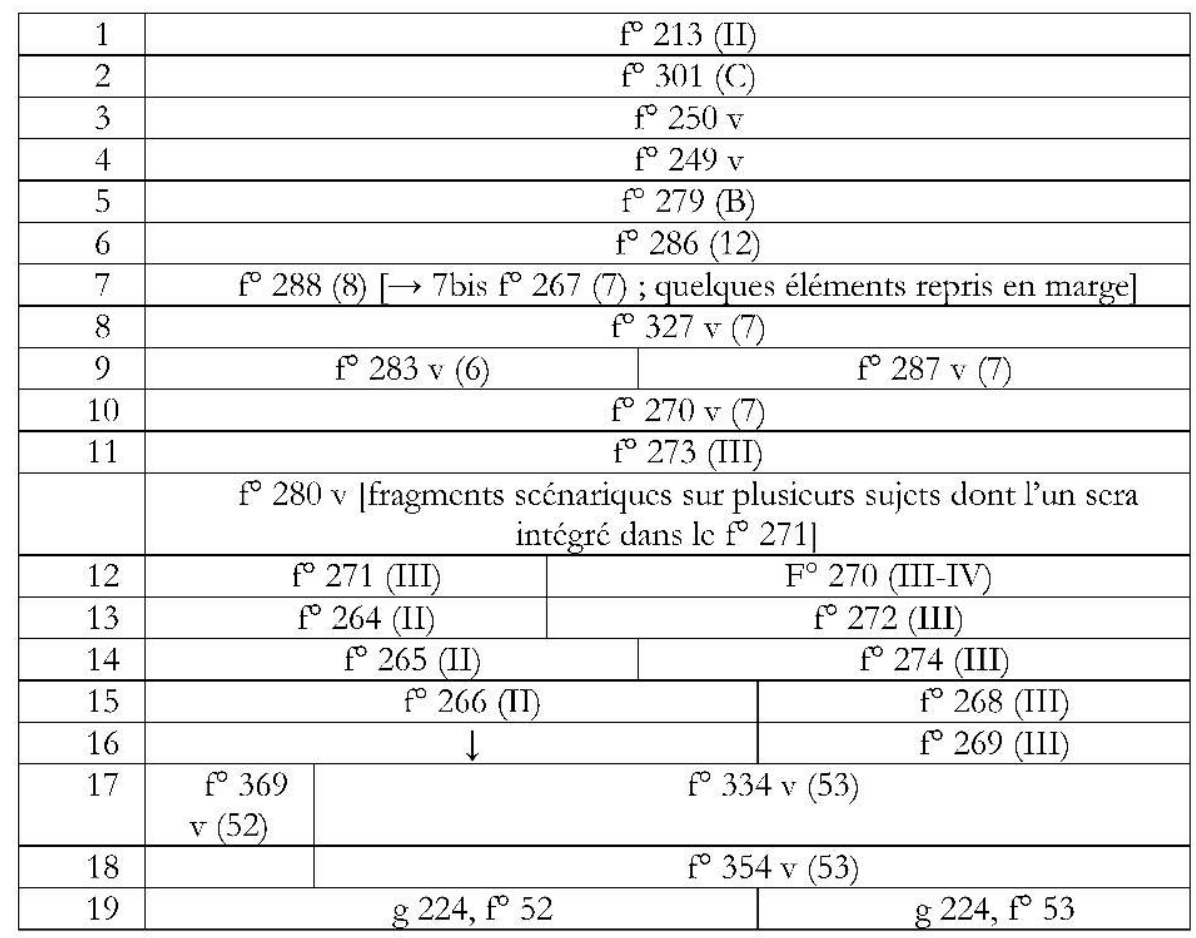

L'idée des deux expériences menées parallèlement n'a pas été trouvée dès le début. Dans la version que nous avons citée précédemment et qui est en vérité la cinquième occurrence de l'épisode, la conjugaison du verbe («restent») suggère que les deux bonshommes pratiquent simultanément l'expérience de la perspiration, bien qu'il soit difficile de les imaginer debout tous deux sur une balance! Quant à l'autre expérience, celle de la contraction musculaire, l'occurrence suivante spécifie dans le même sens : « ils se mirent dans leur baignoire un bain à peine tiède " ( $\left.f^{\circ} 286\right)$. On peut en conclure qu'à ce stade, Bouvard et Pécuchet devaient se livrer successivement à ces deux expériences qu'ils exécutaient ensemble à chaque fois. Toutefois, on voit apparaitre en même temps 
un petit changement qui affecte d'abord l'expérience de la balance : « Pour voir si le corps poids du corps diminue de poids par la perspiration, ils se placèrent à tour de rôle sur leurs balances » (fo 286). Aussi n'est-ce plus simultanément, mais successivement qu'ils se mettent sur la balance, ce qui les conduit à diviser les tâches : «L'un se tenait tranquille immobile sur le plateau de la balance tandis que l'autre, une montre à la main devait observer les changements » (fo 286). On peut alors s'attendre à une modification similaire de l'autre expérience, ce qui advient dans la version suivante où l'adverbe " alternativement » est inséré après le verbe pronominal : «ils se mirent alternativement dans un bain à peine tiède $[. .] ».\left(f^{\circ} 288\right)$.

19 La structuration du récit étant arrivée à ce point, l'écrivain commence à s'occuper de la production des tableaux et note en marge cette consigne : «développer l'image » ( $\left.f^{\circ} 288\right)$. La notation se trouve en face de l'épisode de la perspiration, mais on peut la rapporter, sans grand risque de contresens, à l'ensemble des deux expériences. De surcroît, c'est fort probablement cette préoccupation des images qui amène maintenant l'écrivain à assigner une expérience précise à chacun des protagonistes. Le corps maigre de Pécuchet sera exhibé nu sur la balance, alors que Bouvard pratiquera un exercice vif et cocasse en remuant son corps volumineux. Le contraste est on ne peut plus comique. Cette distribution des rôles, en tout cas, sera fixée dans la huitième occurrence. Au fo $327 \mathrm{v}^{\circ}$, Flaubert écrit d'abord: "ils se mirent dans un bain à peine tiède", et continue le paragraphe à la troisième personne du pluriel ("tachèrent", "grelottaient", " claquaient », « leur gymnastique »). Et puis, après un moment d'hésitation que souligne l'adverbe « alternativement » repris en interligne avant d'être barré aussitôt, il décide de remplacer le pronom «ils» par «Bouvard». Un pas capital est franchi, et Flaubert attribue naturellement à Pécuchet le paragraphe suivant consacré à la perspiration. Cela lui permet aussi d'inventer un détail qui se révélera thématiquement fort riche comme nous le verrons plus bas : "Pécuchet hésite à se déshabiller». Les deux bonshommes assument dès lors chacun l'expérience qui est la mieux adaptée à la virtualité comique de son corps. Certes, il y a encore à cette étape très peu d'éléments descriptifs ( "grosse [sic] ventre » et « cachalot » à propos de Bouvard), mais la note de régie (« son portrait»), qui se retrouve à l'identique dans les deux paragraphes, atteste la volonté de l'écrivain de profiter au mieux de cette plasticité virtuelle. Cela aboutira, on le sait, à deux morceaux grotesques dans la version finale: "et il se tenait sur le plateau, complètement nu, laissant voir, malgré la pudeur, son torse très long pareil à un cylindre, avec des jambes courtes, les pieds plats et la peau brune »; "Bouvard ouvrit les cuisses, se tordait les flancs, balançait son ventre, soufflait comme un cachalot » (p. 115) ${ }^{36}$.

Notons que les deux expériences étaient placées jusqu'ici dans l'ordre inverse du texte publié. Il en est de même dans la version suivante (neuvième occurrence), formée par deux folios qui font partie d'une même séquence génétique ( $f^{\circ} 283 v^{\circ}$ et $f^{\circ} 287 v^{\circ}$ ). La fin $\mathrm{du}$ premier folio consacrée à la contraction musculaire s'enchaîne avec le début $d u$ second portant sur la perspiration insensible. Cependant, on voit intervenir à cette étape une suite de remaniements décisifs qui transformeront soudainement la structure du passage et resserreront le tissu narratif. Premièrement, Flaubert s'avise de terminer la scène de la balance par l'entrée du chien : «à la fin du pesage (un chien le décrire) entre par hasard dans le fournil - Si nous le gardions pour les expériences " ( $\left.f^{\circ} 287 \mathrm{v}^{\circ}\right)$. Idée fructueuse dans la mesure où elle met en place une transition avec l'épisode suivant dans lequel sera mise en danger la vie de l'animal. En même temps, cet ajout interlinéaire semble avoir poussé Flaubert à réviser l'organisation de l'épisode entier, comme en témoigne cette 
notation inscrite dans la marge du fo $283 \mathrm{v}^{\circ}:$ « d'abord P. sur la balance. / - puis B. dans sa baign[oire] / entrée du chien. » Enfin, l'idée vient au romancier d'accentuer l'effet de comique visuel de la scène en mettant à la fois les deux corps romanesques sur la scène. C'est ainsi qu'il note dans la marge du fo $287 \mathrm{v}^{\circ}$ : « les 2 expér[iences] simultanées ».

21 La balance d'abord, le bain ensuite. Cet ordre nouveau, que Flaubert ne modifiera plus, a de surcroît le mérite de résoudre une difficulté qui demeurait jusque-là sans solution: que fait Bouvard pendant que Pécuchet reste debout sur la balance ? La version proposée par le texte de premier jet au fo $287 \mathrm{v}^{\circ}$ («B. pendant ce temps-là, se promenait ou lui faisait la lecture ») témoigne plutôt d'un embarras de l'écrivain qui ne parvient pas à lier de façon organique tous les éléments du récit. Ces comportements de Bouvard (promenade et lecture) demeurent encore à ce stade essentiellement oiseux, en ce sens qu'ils sont dépourvus de fonctionnalité narrative. Au contraire, en inversant l'ordre des deux expériences, l'écrivain se trouve désormais en mesure d'utiliser «la lecture » de Bouvard comme transition entre elles. Mais comme il arrive parfois dans la genèse flaubertienne, il reste momentanément aveugle à cette possibilité d'enchaînement, dont il s'apercevra plus tard, lors de la relecture de la douzième version. Nous citons ici, pour des raisons de lisibilité, la treizième version où l'on trouve intégrés dans le corps du texte les ajouts interlinéaires de la version précédente : «[...] \& assis sur une chaise escapeau [sic] lui faisait la lecture - / à propos de la chaleur animale, Richerand affirme que ils apprirent que la ch[aleur] anim[ale] se développe par les contractions mus[culaires] » (f 272$)$.

Il faut relever un autre effet, non moins important, de cette inversion de l'ordre. L'expérience de la perspiration faisant dorénavant directement suite à l'épisode de la génération, la mention de la pudeur de Pécuchet sur la balance se trouve rapprochée de l'aveu de sa virginité qui l'explique en retour. On voit ainsi mis en valeur un réseau thématique qui traverse en diagonale l'ensemble du roman ${ }^{37}$. Sur ce point, il est intéressant de remarquer que l'invention de l'épisode de l'aveu se fait au même moment que le remaniement de l'organisation narrative. C'est en effet sur le fo $283 \mathrm{v}^{\circ}$, où Flaubert change l'ordre des expériences, que l'on peut lire cet ajout interlinéaire au crayon: " génération - aveu de Pécuchet ». Si les manuscrits antérieurs prévoyaient déjà de traiter de la génération, il s'agissait là plutôt de problèmes théoriques comme celui de l'ovulation. La courte notation ajoutée au fo $283 \mathrm{v}^{\circ}$ a pour effet de centrer l'épisode de la génération sur l'ignorance de Pécuchet en cette matière et sur son aveu de pucelage, et du coup, instaure un lien entre deux épisodes qui se succèdent. Quant à l'hésitation de Pécuchet à se mettre nu, dont on a déjà vu la première apparition $\left(f^{\circ} 327 \mathrm{v}^{\circ}\right)$, elle provoque d'abord l'irritation de son ami comme dans la onzième occurrence : "Vas-tu recommencer tes bêtises, allons donc! » (f' 273$)$; ou dans la douzième : « Finis donc tes bêtises! dit Bouvard. Entre hom [mes] pas de pudeur! " (fo 270). Flaubert ne tardera pas à abandonner ce petit dialogue, qui a sans doute l'inconvénient de rendre trop manifeste le lien entre la virginité de Pécuchet et sa pudeur. En revanche, le mot «pudeur », qui apparaît pour la première fois au fo 270 , subsistera jusqu'au texte final. L'application de ce mot à l'un des deux bonshommes est d'un comique indéniable, car la pudeur a été longtemps considérée comme un attribut de la féminité38. Le Dictionnaire des idées reçues la définit comme «le plus bel ornement de la femme $»^{39}$. Attribuée à Pécuchet en train de se peser nu sur le plateau, elle ajoute un trait supplémentaire à la picturalité de son geste expérimental. Seulement, le mot devra être transféré dans un autre contexte, celui de la description de sa posture. Flaubert procède à cette opération dans la marge du fo 272 (treizième 
occurrence) : « Aalgré sa pudeur Malgré sa peur du rire* il retira il ôta ses habits [en] dépit de la pudeur et se tenait debout sur le plateau complèt[ement] nu $-»^{40}$.

Les exemples que nous avons analysés montrent à quel point la mise en texte des savoirs dans Bouvard et Pécuchet est le fruit d'un travail long et ardu de l'écrivain. Le dialogue et le tableau lui offrent deux dispositifs principaux aptes à intégrer le cognitif dans la fiction romanesque. Ancré profondément dans l'horizon des connaissances du XIX ${ }^{e}$ siècle, le roman encyclopédique nous donne à voir les rêves, les illusions et les insuffisances qui marquaient les savoirs de l'époque. En ce sens, l'imaginaire des documents qu'il libère est incontestablement daté. Il est tout à fait vraisemblable que de nombreuses contradictions pointées par notre romancier n'apparaissent plus comme telles pour la pensée scientifique de nos jours. Ce constat ne revient pourtant pas à dénier la portée critique de Bouvard, qui reste parfaitement valable en dépit du recul progressif des limites des connaissances humaines. Car ce que la représentation romanesque de Flaubert met en évidence, ce n'est pas, à vrai dire, la fragilité de telle ou telle théorie ou hypothèse, mais plus fondamentalement la posture même de la pensée qui prétend avoir l'exclusivité de la vérité. C'est pourquoi la visualité est un facteur essentiel de son texte, remarquable surtout par sa capacité singulière d'exposition ${ }^{41}$. Flaubert s'attache avant tout à saisir le comique qui réside dans l'acte même d'affirmation, dont il défait la pesanteur en l'exposant. Dans l'univers du roman encyclopédique, toute volonté de savoir est à la limite ramenée à cette question du geste, et par là, interrogée dans sa dimension proprement anthropologique. C'est là, somme toute, ce que Flaubert appelait «le comique d'idées ", qui ne cesse de nous intriguer par sa modernité fondamentale.

\section{NOTES}

1. Correspondance, édition de Jean Bruneau et d'Yvan Leclerc (pour le tome V), Gallimard, « Bibliothèque de la Pléiade », t. II, p. 519.

2. Sur ce point voir notre article : «La littérature comme négativité : Flaubert et Sartre », Bulletin de la section française, Faculté des Lettres, Université Rikkyo, n 40, 2011, p. 87-91.

3. URL : http://flaubert.univ-rouen.fr/bouvard_et_pecuchet/chantier/roman_final/ BPframe1.html (responsable : Yvan Leclerc).

4. URL : http://dossiers-flaubert.ish-lyon.cnrs.fr/ (responsable: Stéphanie Dord-Crouslé).

5. Les citations du texte de Bouvard et Pécuchet renvoient à l'édition mise à jour de Stéphanie Dord-Crouslé, Flammarion, « GF », 2008.

6. On a souvent remarqué, et à juste titre, les incohérences relatives à la chronologie du roman. Cependant Flaubert ne se montrait pas toujours indifférent au temps représenté de l'histoire des deux bonshommes. En témoigne par exemple cette lettre à Maxime Du Camp du 14 mars 1877 : "Possèdes-tu quelque bouquin de physiologie imbécile? Il faut que l'ouvrage ait au moins quarante ans de date!» (Correspondance, op.cit., t. V, p. 203). Les ouvrages de Richerand et d'Adelon répondent bien à cette préoccupation réaliste.

7. J. Neefs, «L'imaginaire des documents », dans Romans d'archives, textes réunis et présentés par Raymonde Debray-Genette et Jacques Neefs, Lille, Presses Universitaires de Lille, 1987, p. 175-189.

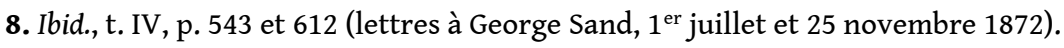


9. Ibid., t. V, p. 214 (lettre à Edma Roger des Genettes, 2 avril 1877).

10. M. Du Camp, Souvenirs littéraires, préface de Daniel Oster, Aubier, 1994, p. 618.

11. Correspondance, op. cit., t. IV, p. 559 (lettre à E. Roger des Genettes, 19 août 1872).

12. Ibid., t. V, p. 214 (lettre à la même, 2 avril 1877).

13. Ibid., t. II, p. 71 (lettre à L. Colet, 15 avril 1852).

14. Ibid., t. V, p. 796 (lettre à E. Roger des Genettes, 24 janvier 1880).

15. Ibid., t. IV, p. 1000 (lettre à G. Sand, fin décembre 1875).

16. L'expression se trouve dans plusieurs brouillons, comme au g $226^{3}$, fo $249 \mathrm{v}$ : « impasse de la physiologie : la Nutrition " .

17. Dans le cas de la nutrition, c'est le traité d'Adelon déjà cité qui a servi de source documentaire.

18. Sur cette dispute et sa densité épistémologique remarquable, voir le chapitre VI de notre ouvrage Flaubert épistémologue. Autour du dossier médical de Bouvard et Pécuchet, Amsterdam - New York, Rodopi, coll. « Faux titre », 2010.

19. Le dossier des notes médicales contient aussi deux pages de «notes de notes » dans lesquelles on voit traitée la question des pulsations : $g$ 226 $6^{7}$, 138 et $\mathrm{f}^{\circ} 139 \mathrm{v}^{\circ}$. Ces pages dont le caractère scénarique est évident doivent être chronologiquement placées avant le premier brouillon.

20. Pour les citations des brouillons appartenant tous au volume g $225^{3}$, nous donnerons seulement le numéro de folio.

21. Comme on le verra plus bas, dans les premières versions se trouvent cités les noms de Capuron, Tissot et Buchan dont le dernier est ensuite remplacé par « la pathologie d'Andral ».

22. Flaubert a noté en marge : "Contrad[iction] ».

23. Le mode de transcription est diplomatique. Néanmoins, nous avons choisi, pour la commodité de présentation, d'incorporer dans le corps du texte les ajouts interlinéaires marqués par l'italique.

24. L'extrait se trouve enregistré dans les notes sur la Physiologie de l'homme ( $\mathrm{g} 226^{7}$, $\mathrm{f}^{\circ}$ 69).

25. Correspondance, op.cit., t. V, p. 482 (lettre à Léonie Brainne, 30 décembre 1878). Dans un scénario, Flaubert envisageait de «donner comme vraies des indications bibliographiques fausses » (gg 10, $\left.\mathrm{f}^{\circ} 5\right)$.

26. Les ouvrages de ces auteurs sur lesquels Flaubert a pris des notes sont: Joseph Capuron, Manuel des Dames de charité, Paris, Thomine, Leriche, 1816 ; Samuel Tissot, Avis au peuple sur sa santé, (Lausanne, F. Grasset, 1761) ; William Buchan, Médecine domestique, $5^{\mathrm{e}}$ édition, Paris, Moutardier, 1802, 5 vol. L'édition de l'ouvrage de Tissot utilisée par Flaubert est inconnue.

27. Le dernier numéro de la série Gustave Flaubert $\left(\mathrm{n}^{\circ} 7\right.$, textes réunis et présentés par Gisèle Séginger, Caen, Minard, "La Revue des lettres modernes", 2010) consacré à «Flaubert et la peinture » contient plusieurs articles qui traitent de la caractéristique des tableaux flaubertiens. L'introduction par G. Séginger propose une mise au point claire et très utile.

28. Correspondance, op. cit., t. I, p. 680 (lettre à Louis Bouilhet, 4 septembre 1850).

29. Ibid., t. II, p. 513 (lettre à L. Colet, 23 janvier 1854).

30. Sur la note de régie chez Flaubert, voir Yvan Leclerc, « Les notes de régie dans les manuscrits de Madame Bovary. Flaubert et le savoir du roman ", dans Madame Bovary et les savoirs, Pierre-Louis Rey et Gisèle Séginger (éds), Presses Sorbonne nouvelle, 2009, p. 23-34.

31. Ibid., t. II, p. 298 (lettre à L. Colet, 6 avril 1853).

32. Ibid., t. V, p. 213-214 (lettre à E. Roger des Genettes, 2 avril 1877).

33. Ibid. Anne Herschberg Pierrot a analysé l'implication sémantique de cette formule célèbre dans son article sur "La mise en texte des savoirs dans Bouvard et Pécuchet », dans La mise en texte des savoirs, textes réunis par Kazuhiro Matsuzawa et Gisèle Séginger, Presses universitaires de Strasbourg, 2010, p. 321-331.

34. Les mentions «Lévy " et "Béraud » renvoient respectivement au Traité d'hygiène publique et privée de Michel Lévy (Paris, J.-B. Baillière et fils, 1844-45, 2 vol.) et aux Éléments de physiologie de 
l'homme de Bruno Béraud ( $2^{\mathrm{e}}$ édition, Paris, G. Baillière, 1856-57, 2 vol.). Outre ces références données ici par Flaubert, le traité de Richerand dont on a déjà parlé et l'Histoire des sciences médicales de Charles Daremberg (Paris, J.-B. Baillière et fils, 1870, 2 vol.) font partie de l'intertexte de l'épisode. Voir à ce sujet notre ouvrage déjà cité, p. 99-102.

35. Nous mettons entre parenthèses les chiffres écrits par Flaubert en haut à droite des folios. On trouve aussi dans le dossier médical des «notes de notes" qui comprennent des fragments concernant les deux expériences en question : $\mathrm{g} 226^{7}, \mathrm{f}^{\circ} 143,144,144 \mathrm{v}^{\circ}$.

36. Dans le cadre de cet article, nous ne pouvons pas suivre de près la production de ces morceaux descriptifs. Nous tenons pourtant à signaler que la description de Pécuchet a connu des versions très amplifiées au cours de la rédaction. Voici, par exemple, la transcription diplomatique de la treizième occurrence que nous linéarisons autant que possible : «Enfin il exhiba son laissant voir son torse très long maigre de forme cylindrique avec des salières sous les clav [icules] / sur à des jambes trop courtes \& un peu cagneuses avec des bras minces / la peau bronzée, bistrée brune rousse, |des salières aux dans les clavicules| les côtes saillantes \& au bas milieu du ventre l'abdo[men] / une tache de vin lun de ces nævus violets, qu'on nommé tête de hareng, une envie de sa mère / quand elle était grosse de lui| » ( $\left.f^{\circ} 272\right)$.

37. Dès sa première rencontre avec Bouvard sur le boulevard Bourdon, Pécuchet fait preuve de timidité à l'égard du fait sexuel. Ainsi, face à son ami qui «se permit une réflexion obscène " à propos d'une fille de joie, «Pécuchet devint très rouge, et sans doute pour s'éviter de répondre, lui désigna du regard un prêtre qui s'avançait » (p. 47).

38. Voir sur ce point Jean-Claude Bologne, Histoire de la pudeur, Hachette Littératures, "Pluriel », 1997.

39. Bouvard et Pécuchet, édition de Claudine Gothot-Mersch, Gallimard, «folio », 1979, p. 548. L'article ne figure pas dans la dernière version du Dictionnaire.

40. Le signe * indique une lecture conjecturale.

41. Stéphanie Dord-Crouslé évoque très pertinemment l'importance des corps des personnages même dans les scènes du dialogue. Le langage du corps redoublant l'affrontement des arguments et des doctrines procure une visibilité accrue à la scène. "Le débat est alors reproduit dans l'ordre du corps. » Voir Bouvard et Pécuchet de Flaubert. Une "encyclopédie critique en farce ", Belin, «Lettres Sup », 2000, p. 53-56.

INDEX

Mots-clés : savoir, documentation, science, travail scénarique

\author{
AUTEUR
}

NORIOKI SUGAYA 